\title{
Differential pathlength spectroscopy for diagnosis of head and neck
}

\section{cancer}

\author{
Henricus JCM Sterenborg*, Max JH Witjes, Sebastiaan De Visscher and \\ Arjen Amelink
}

Address: Center for Optical Diagnostics and Therapy, Erasmus Medical Centre Rotterdam, The Netherlands

* Corresponding author

from Ist Scientific Meeting of the Head and Neck Optical Diagnostics Society

London, UK. I 4 March 2009

Published: 28 July 2009

Head \& Neck Oncology 2009, I(Suppl I):O7 doi:I0.I I86/I758-3284-I-SI-O7

This abstract is available from: http://www.headandneckoncology.org/content/I/SI/O7

(C) 2009 Sterenborg et al; licensee BioMed Central Ltd.

The development of optical techniques for non-invasive diagnosis of cancer is an ongoing challenge to biomedical optics. For head and neck cancer we see two main fields of potential application

1) Screening for second primaries in patients with a history of oral cancer. This requires imaging techniques or an approach where a larger area can be scanned quickly.

2) Distinguishing potentially malignant visible primary lesions from benign ones. Here fiberoptic point measurements can be used as the location of the lesion is known.

This presentation will focus on point measurement techniques.

Various techniques for point measurements have been developed and investigated clinically for different applications. Differential Pathlength Spectroscopy is a recently developed fiberoptic point measurement technique that measures scattered light in a broad spectrum. Due to the specific fiberoptic geometry we measure only scattered photons that have travelled a predetermined pathlength. This allows us to analyse the spectrum mathematically and translate the measured curve into a set of parameters that are related to the microvasculature and to the intracellular morphology. DPS has been extensively evaluated on optical phantoms and tested clinically in various clinical applications.
The first measurements in biopsy proven squamous cell carcinoma showed significant changes in both vascular and morphological parameters. Measurements on thick keratinized lesions however failed to generate any vascular signatures. This is related to the sampling depth of the standard optical fibers used. Recently we developed a fiberoptic probe with a $\sim 1 \mathrm{~mm}$ sampling depth. Measurements on several leukoplakias showed that with this new probe we sample just below the keratin layer and can obtain vascular signatures. The results of a first set of clinical measurements will be presented and the significance for clinical diagnostics will be discussed. 